\title{
Direct interaction between caffeic acid phenethyl ester and human neutrophil elastase inhibits the growth and migration of PANC-1 cells
}

\author{
JIANHUI DUAN, YILIXIATI XIAOKAITI, SHENGJUN FAN, YAN PAN, XIN LI and XUEJUN LI \\ Department of Pharmacology, School of Basic Medical Sciences, Peking University and State Key Laboratory \\ of Natural and Biomimetic Drugs, Peking University and Beijing Key Laboratory of \\ Tumor Systems Biology, Peking University, Beijing 100191, P.R. China
}

Received November 17, 2016; Accepted January 16, 2017

DOI: 10.3892/or.2017.5516

\begin{abstract}
Pancreatic ductal adenocarcinoma (PDAC) is one of the most lethal malignant tumors of the digestive system, but the mechanisms of its development and progression are unclear. Inflammation is thought to be fundamental to pancreatic cancer development and caffeic acid phenethyl ester (CAPE) is an active component of honey bee resin or propolis with anti-inflammatory and anticancer activities. We investigated the inhibitory effects of CAPE on cell growth and migration induced by human neutrophil elastase (HNE) and report that HNE induced cancer cell migration at low doses and growth at higher doses. In contrast, lower CAPE doses inhibited migration and higher doses of CAPE inhibited the growth induced by HNE. HNE activity was significantly inhibited by CAPE (7.5-120 $\mu \mathrm{M})$. Using quantitative real-time PCR and western blotting, we observed that CAPE (18-60 $\mu \mathrm{M})$ did not affect transcription and translation of $\alpha 1$-antitrypsin ( $\alpha 1-\mathrm{AT})$, an endogenous HNE inhibitor. However, in an in silico drug target docking model, we found that CAPE directly bound to the binding pocket of HNE $(25.66 \mathrm{kcal} / \mathrm{mol})$ according to CDOCKER, and the residue of the catalytic site stabilized the interaction between CAPE and HNE as evidenced by molecular dynamic simulation. Response unit (RU) values of surface plasmon resonance (SPR) significantly increased with incremental CAPE doses (7.5-120 $\mu \mathrm{M})$, indicating that CAPE could directly bind to HNE in a concentration-dependent manner. Thus, CAPE is an effective inhibitor of HNE via direct interaction whereby it inhibits the migration and growth of PANC-1 cells in a dose-dependent manner.
\end{abstract}

Correspondence to: Professor Xuejun Li, Department of Pharmacology, School of Basic Medical Sciences, Peking University, 38 Xueyuan Road, Beijing 100191, P.R. China

E-mail: xjli@bjmu.edu.cn

Key words: caffeic acid phenethyl ester, human neutrophil elastase, migration, growth

\section{Introduction}

Pancreatic ductal adenocarcinoma (PDAC) is one of the most lethal and malignant tumors of the digestive system. The rate of successful PDAC resection is less than $20 \%$ and most patients present with disease recurrence. The 5-year survival rate of PDAC is only $~ 5 \%$ despite therapeutic advances in diagnosis and treatment (1). PDAC is the fourth leading cause of cancerrelated deaths in the US with an estimate of 37,390 deaths closely following the number of 43,920 diagnoses in 2012 (2) and an estimate of 38,460 deaths closely following the number of 45,220 diagnoses in 2013 (3). Moreover, PDAC has been projected to advance as one of the leading 3 cancer killers in 2030 (4). PDAC is characterized by infiltration of polymorphonuclear neutrophils (PMNs) into the desmoplastic stroma in most cancer samples. PMN infiltration may create a proinflammatory microenvironment affecting tumor progression by diverse mechanisms $(5,6)$.

PMNs consist of various enzymes, including human neutrophil elastase (HNE) $(7,8)$. HNE is a neutral protease of the serine protease superfamily and is chiefly stored in neutrophilic granulocytes. In addition, various cancer cells contain $\operatorname{HNE}(9,10)$. Normally, HNE does not injure cells, but HNE may have an important regulatory role in local inflammatory responses and dysregulation causing HNE to be accumulated and produce chronic inflammatory disease (11). Inflammation contributes to numerous cancers such as lung, bladder, gastrointestinal tract, skin and vulva cancer (12). Inflammation may promote cancer growth, tissue invasion and metastasis, characterized by reactive oxygen species and cell cycling induction. Cancer arising from inflammation may be associated with a worse prognosis (13). $\alpha 1$-antitrypsin, an endogenous inhibitor of HNE, is implicated in $\alpha 1$-antitrypsin-HNE imbalances and this is thought to create a favorable tissue environment for carcinogens and tumor progression, promoting the growth, survival, metastasis of tumor cells $(11,14)$. Inhibition of HNE by its specific inhibitor was found to significantly suppress growth, motility and chemotaxis of the pancreatic carcinoma cell line Capan-1 (15).

Caffeic acid phenethyl ester (CAPE) is an active component of the honey bee resin, propolis, and is reported to have anti-inflammatory, neuroprotective, hepatoprotective, 
cardioprotective, immunomodulatory and anticancer effects $(16,17)$. The tumor-specific cytotoxicity of CAPE has been reported as CAPE can inhibit tumor cell growth, infiltration and metastasis in vivo and in vitro, including central nervous system, gastrointestinal system and breast cancers as well as leukemias $(18,19)$. CAPE modulates the inflammatory response by reducing $\mathrm{c}$-jun-N-terminal kinase and nuclear factor $(\mathrm{NK})-\kappa \mathrm{B}$ activation and decreasing cyclooxygenase (COX)-2 expression (16). However, how this occurs is unclear. Thus, we investigated the contribution of inflammation to pancreatic cancer progression and the novel effects of CAPE on HNE, as well as the interaction between CAPE and HNE.

\section{Materials and methods}

Cell culture and treatment. The PDAC PANC-1 cell line was used for in vitro experiments. Cells were maintained in Dulbecco's modified essential medium (DMEM) plus 10\% fetal bovine serum (FBS) (both from Gibco, Grand Island, $\mathrm{NY}, \mathrm{USA}$ ), $100 \mathrm{U} / \mathrm{ml}$ penicillin and $100 \mathrm{mg} / \mathrm{ml}$ streptomycin in a humidified incubator with $5 \% \mathrm{CO}_{2}$ in air at $37^{\circ} \mathrm{C}$. Cells were treated with different concentrations of HNE (Merck Group, Darmstadt, Germany) and/or CAPE (R\&D Systems/Tocris, Minneapolis, MN, USA) for 24 or $48 \mathrm{~h}$, and then evaluated.

Cell viability assay. Cytotoxicity was measured using the CellTiter $96^{\circledR} \mathrm{AQ}_{\text {ueous }}$ One Solution Cell Proliferation Assay kit (Promega, Madison, WI, USA). Briefly, PANC-1 cells growing in log-phase were trypsinized and seeded at 5,000 cells/well into 96-well plates, and allowed to adhere for $24 \mathrm{~h}$. Media were replaced by fresh medium or medium with HNE and/or CAPE, and incubated for $48 \mathrm{~h}$. One-fifth of the volume of CellTiter $96^{\circledR} \mathrm{AQ}_{\text {ueous }}$ One solution was added to each well and incubated for an additional $3 \mathrm{~h}$. The resulting color was assayed at $490 \mathrm{~nm}$ using a microplate reader (Bio-Rad Laboratories, Inc., Hercules, CA, USA). Blank control wells were used for zeroing the absorbance.

Wound-healing assay of PANC-1 cell migration. PANC-1 cells stably transfected with GFP were seeded into 6-well plates. Confluent monolayers were scratched using a sterile $1,000-\mu 1$ pipette tip to create a uniform cell-free zone in each well. Suspended cells were removed by washing with normal growth medium. Wounded monolayers were then incubated in the presence or absence of $10 \mathrm{nM}$ HNE and decreasing concentrations of CAPE $(27 \mu \mathrm{M}$ with 1.5 -fold dilutions to $8 \mu \mathrm{M})$. A scratch wound was captured after 24 or $48 \mathrm{~h}$ using an Olympus microscope in 3 fields of view at a magnification of $x 40$. The recovered area was measured using PhotoShop software.

Elastase assay. HNE activity was measured with an enzyme assay in 96-multiwell plates. Briefly, $50 \mu \mathrm{l}$ substrate solution (1.4 mM MeO-Suc-Ala-Ala-Pro-Val-pNA in Tris-HCl buffer, $\mathrm{pH} 7.5$ ) was mixed with $140 \mu 1$ test solution (test substances solubilized in Tris- $\mathrm{HCl}$ buffer, $\mathrm{pH}$ 7.5) and vortexed. After the addition of $10 \mu \mathrm{l} \mathrm{HNE}$ solution, the samples were incubated for $1 \mathrm{~h}$ at $37^{\circ} \mathrm{C}$. The reaction was stopped by the addition of $200 \mu \mathrm{l}$ soybean trypsin inhibitor solution $(2 \mathrm{mg} / \mathrm{ml}$ Tris- $\mathrm{HCl}$ buffer, $\mathrm{pH}$ 7.5) and placed in an ice bath. After vortexing, the absor- bance was read at $405 \mathrm{~nm}$. Inhibition rates were calculated as a percentage of the controls without inhibitors (20). Sivelestat (Siv) sodium was added as a positive control.

Western blotting. Total protein was extracted using RIPA lysis (Applygen Technologies, Inc., Beijing, China) buffer from PANC-1 cell lysates after co-incubation of $40 \mathrm{nM}$ HNE and CAPE (18-60 $\mu \mathrm{M})$ for $48 \mathrm{~h}$. After centrifuging and boiling at $100^{\circ} \mathrm{C}$ for $5 \mathrm{~min}$, equal amounts of proteins were separated by $10 \%$ SDS-PAGE and transferred to a polyvinylidene difluoride (PVDF) membrane (Millipore, Billerica, MA, USA) for analysis of $\alpha 1$-antitrypsin (1:500; Santa Cruz Biotechnology, Inc., Santa Cruz, CA, USA) and GAPDH (1:10,000; SigmaAldrich, St. Louis, MO, USA). Slides were fixed and incubated with the primary antibodies overnight at $4{ }^{\circ} \mathrm{C}$ with gentle agitation, followed by secondary antibody reactions with DyLight 800-labeled IgG [1:10,000; Cell Signaling Technology (CST) Danvers, MA, USA)]. Detection was evaluated using the Odyssey ${ }^{\circledR}$ SA Infrared Imaging System.

RNA isolation and quantitative real-time PCR. Total RNA was extracted using TRIzol reagent (Invitrogen, Carlsbad, CA, USA) from PANC-1 cells at $80 \%$ confluence after the co-incubation of $40 \mathrm{nM}$ HNE and CAPE $(18-60 \mu \mathrm{M})$ for $24 \mathrm{~h}$. Then, total RNA was retrotranscribed into first-strand cDNA using the RevertAid First Strand cDNA Synthesis kit (Fermentas, Burlington, ON, Canada). The primers used for quantitative real-time PCR were as follows: $\alpha 1$-antitrypsin (sense primer, 5'-CCCCACCCAGAGTTGCTC-3' and antisense primer, 5'-GGTTAGGTGACAGCGGGTC-3'); GAPDH (sense primer, 5'-GGAGCGAGATCCCTCCAAAAT-3' and antisense primer, 5'-GGCTGTTGTCATACTTCTCATGG-3'). Quantitative real-time PCR was performed using Mx3005P qPCR System (Agilent Technologies, Santa Clara, CA, USA).

Molecular docking and dynamics simulation. To assess the molecular interactions between CAPE and HNE, a molecular docking study was carried out with CDOCKER protocol from Discovery Studio ${ }^{\text {TM }} 2.5$ (DS; Accelrys Software Inc., San Diego, CA, USA). For ligand preparation, a 2-D structure of CAPE was produced by KegDraw software. A force field was applied and binding energy was minimized before the docking procedure. For receptor preparation, the X-ray crystal structure of HNE was obtained from the Protein Data Bank (PDB; http://www.rcsb.org/) with PDB ID: 1B0F. Then, a ligand-based similarity search scheme was employed and the docking protocol was performed as a default setting to avoid a potential reduction in docking accuracy. Molecular dynamic simulations of HNE and CAPE were performed using the standard dynamics cascade protocol in DS. The initial structures of those two simulations were the results of the ligand-receptor complex with the lowest binding energy. The complex was solvated with water in a cubic box with an explicit periodic boundary model to stimulate the environment inside the cell. After creating harmonic restraint, a standard dynamics cascade was performed including minimization, heating, equilibration and production dynamics (21).

Surface plasmon resonance analysis. Surface plasmon resonance (SPR) experiments of CAPE binding to HNE 
A

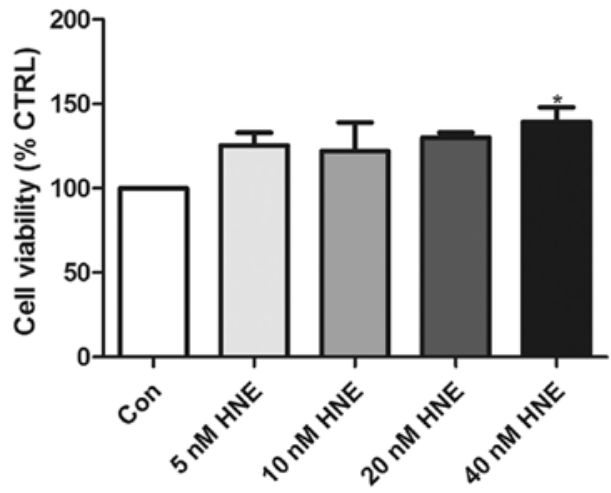

C
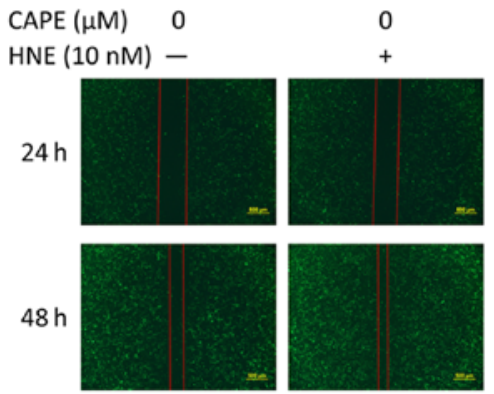

D

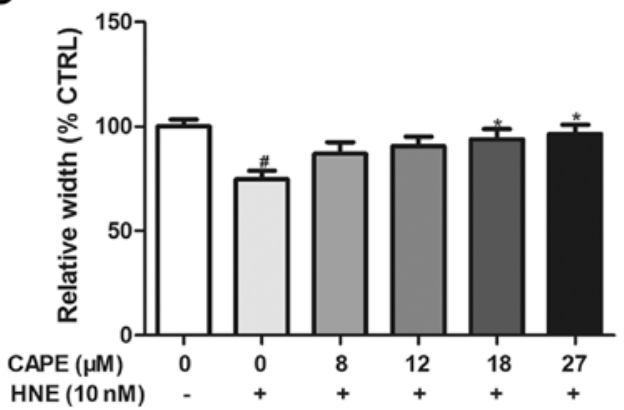

B

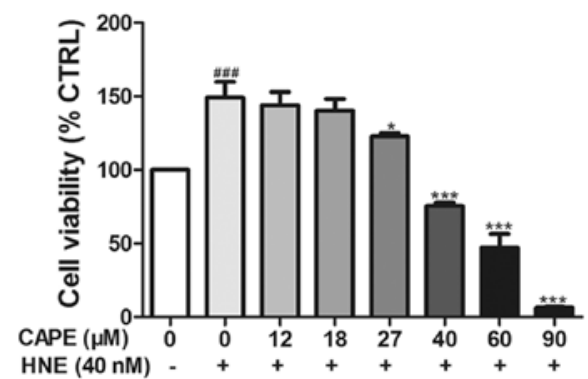

Figure 1. CAPE inhibits PANC-1 tumor cell viability and migration induced by HNE. (A) Cell viability data for PANC-1 cells stimulated with HNE. Data represent means $\pm \mathrm{SEM}, \mathrm{n}=3$; ${ }^{*} \mathrm{P}<0.05$ vs. the HNE-free group (control). (B) Effects of CAPE on the viability of PANC-1 cells treated with $40 \mathrm{nM}$ HNE. (C) The effect of CAPE on cell migration induced by HNE was assessed using a wound-healing method. Values at (D) 24 or (E) $48 \mathrm{~h}$ are expressed as means \pm SEM, $\mathrm{n}=3$ (3 separate experiments); ${ }^{\#} \mathrm{P}<0.05,{ }^{\# \#} \mathrm{P}<0.01,{ }^{\# \# \#} \mathrm{P}<0.001$ compared to the group without $\mathrm{HNE}$ and CAPE (control); $\mathrm{P}<0.05,{ }^{* * * *} \mathrm{P}<0.001$ compared to the group with HNE and without CAPE.

were carried out on a BIAcore ${ }^{\circledR} 3000$ (Biacore AB, Uppsala, Sweden) instrument using Sensor Chip CM5 (GE Healthcare, Fairfield, CT, USA). Pipelines and the chip were pretreated with phosphate-buffered saline (PBS)-ethyl pyruvate (EP) running buffer (10 mM HEPES, $150 \mathrm{mM} \mathrm{NaCl}, 3 \mathrm{mM}$ EDTA, $0.005 \%$ surfactant $\mathrm{P} 20$ and deionized water, $\mathrm{pH}$ 7.4). For SPR analysis, $\sim 500$ resonance units of HNE were immobilized on the CM5 sensor chip using $10 \mathrm{mM}$ sodium acetate buffer with $\mathrm{pH}$ 5.5. Kinetics experiments were carried out by injecting decreasing concentrations of CAPE $(120 \mu \mathrm{M}$ with 2 -fold dilutions to $7.5 \mu \mathrm{M})$ at a flow rate of $10 \mu \mathrm{l} / \mathrm{min}$, and binding responses were recorded for $300 \mathrm{sec}$ in succession. Binding responses were recorded continuously with response unit values. The association $\left(\mathrm{K}_{\mathrm{a}}\right)$ and dissociation $\left(\mathrm{K}_{\mathrm{d}}\right)$ rate constants were elevated by BIA evaluation software (Biacore AB) (21).

Statistical analysis. Significances of the differences between two groups were evaluated by a Student's unpaired t-test and analysis of variance (ANOVA) for comparisons among multiple groups using GraphPad Prism version 5.01 (GraphPad Software, Inc., La Jolla, CA, USA). All data are expressed as mean \pm standard error of measurement (SEM).

\section{Results}

CAPE inhibits tumor cell viability and migration induced by HNE in PANC-1 cells. To measure the cell growth inhibition induced by CAPE in pancreatic cancer cells with HNE in vitro, we measured cell viability in PANC-1 cells using the CellTiter $96^{\circledR} \mathrm{AQ}_{\text {ueous }}$ One Solution cell proliferation assay kit. As shown in Fig. 1A, dose-response curves for the PANC-1 cells indicated that modest concentrations of HNE (10-40 nM) enhanced tumor cell viability, and that $40 \mathrm{nM}$ HNE was the most efficacious, and thus this was used for subsequent experiments. In the presence of $40 \mathrm{nM}$ of HNE, cell viability was significantly decreased with increasing 
A

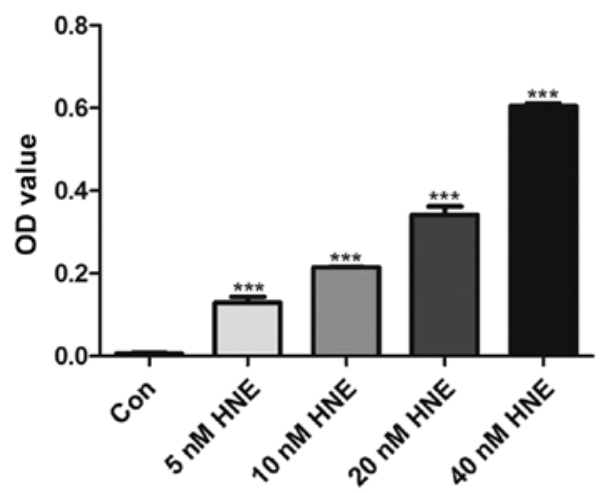

B

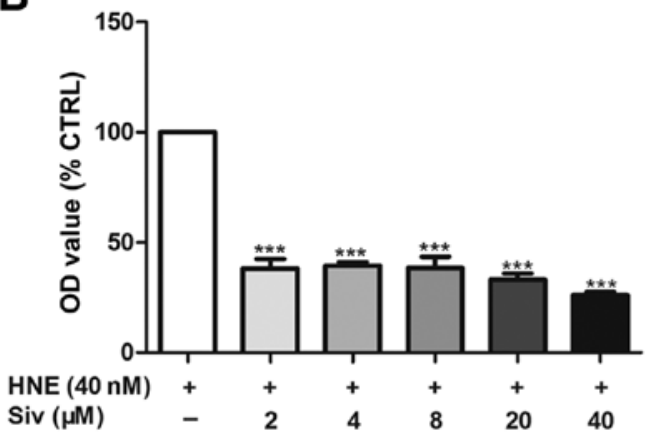

C

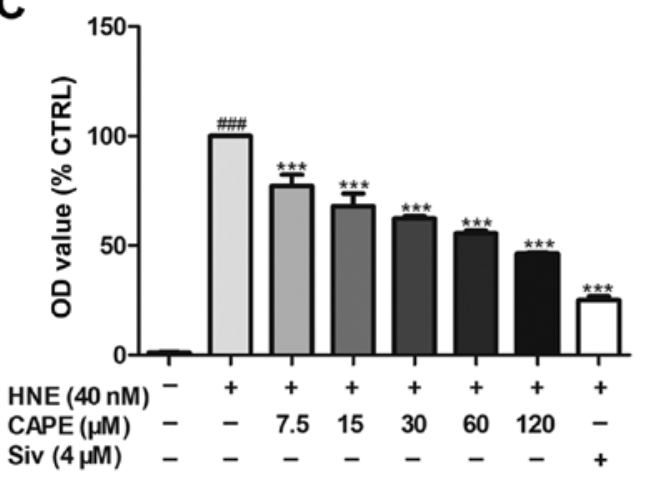

D

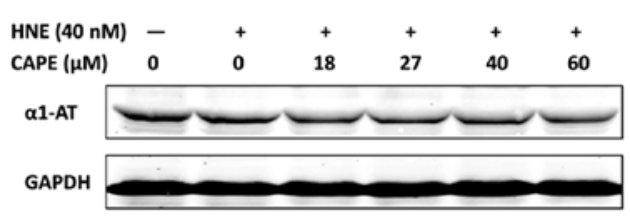

E

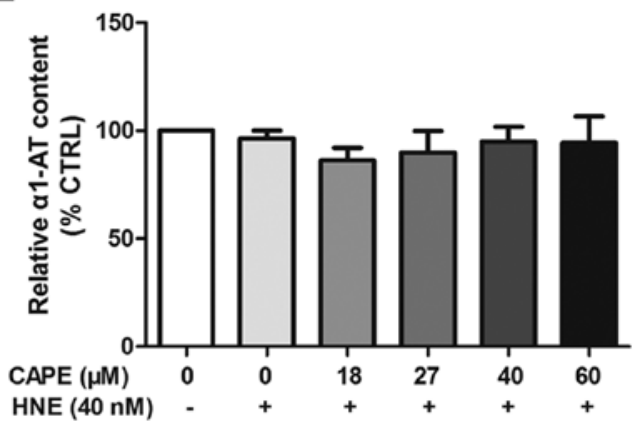

$\mathbf{F}$

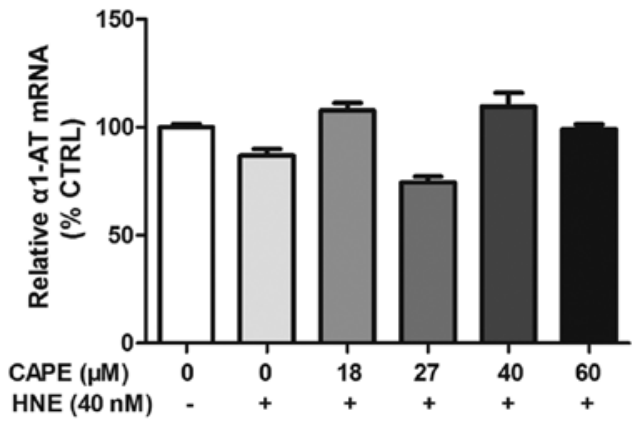

Figure 2. CAPE inhibits HNE activity in an elastase assay, but has no effect on transcription and translation of $\alpha 1$-antitrypsin ( $\alpha 1$-AT). (A) HNE activity. Data represent means $\pm \mathrm{SEM}, \mathrm{n}=3 ;{ }^{* * *} \mathrm{P}<0.001$ compared to HNE-free group (control). (B) The effects of sivelestat (Siv) on HNE activity. Data represent means \pm SEM, $n=3 ;{ }^{* * *} \mathrm{P}<0.001$ compared to the group without sivelestat. (C) Effects of CAPE and Siv on HNE activity. Data represent means \pm SEM,

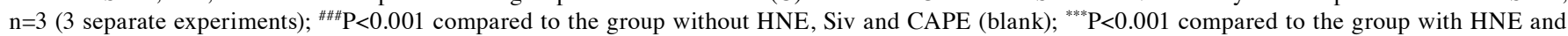
without CAPE and Siv (control). (D and E) Western blotting of $\alpha 1$-antitrypsin in CAPE-treated (18-60 $\mu \mathrm{M})$ and HNE-exposed (40 nM) PANC-1 cell lysates . (F) Quantitative real-time PCR for a1-antitrypsin in PANC-1 cells CAPE-treated (18-60 $\mu \mathrm{M})$ and HNE-exposed (40 nM).

CAPE doses (27-90 $\mu \mathrm{M})$, and lower concentrations had no appreciable effect (Fig. 1B).

To determine whether CAPE inhibits the migration of PANC-1 cells induced by HNE, migration capacity was assessed using an in vitro wound-healing assay into 6-well plates. Fig. 1C-E shows that the migration of PANC-1 cells incubated with $10 \mathrm{nM}$ HNE was increased, whereas incremental doses of CAPE (8-27 $\mu \mathrm{M})$ inhibited the migration of PANC-1 cells. From the cell viability and migration assays, HNE induced cancer cell migration at lower doses, and increased cancer cell viability at higher doses, data that agree with previous research results (22). In addition, our data showed that lower doses of CAPE inhibited migration and higher doses of CAPE inhibited cell growth.
CAPE inhibits HNE activity (elastase assay) in an $\alpha 1$-antitrypsin-independent manner. To study HNE inhibition using an elastase assay, sivelestat sodium, a specific HNE inhibitor, was used as a positive control (Fig. 2B). As shown in Fig. 2A, HNE activity increased in a concentration-dependent manner. In addition, Fig. $2 \mathrm{C}$ shows that HNE activity was also significantly inhibited by CAPE in a concentration-dependent manner $(7.5-120 \mu \mathrm{M})$.

$\alpha 1$-antitrypsin is a serine protease inhibitor (SERPIN) and the endogenous inhibitor of HNE. Several natural products inhibit HNE through the regulation of $\alpha 1$-antitrypsin. To investigate how HNE-induced growth and migration is inhibited by CAPE, western blotting (Fig. 2D and E) and quantitative real-time PCR (Fig. 2F) to determine whether 
A

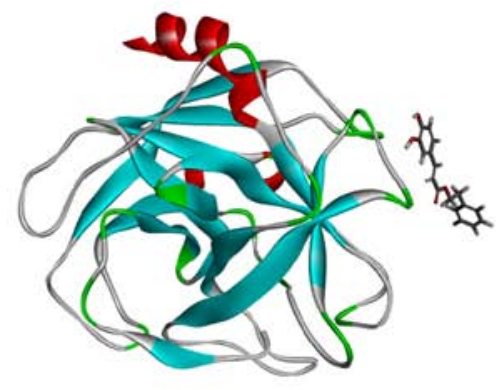

B

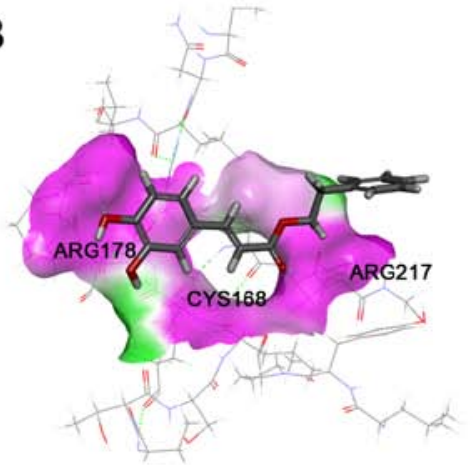

C

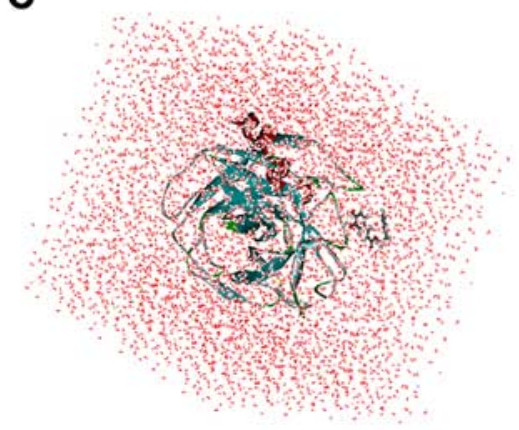

D

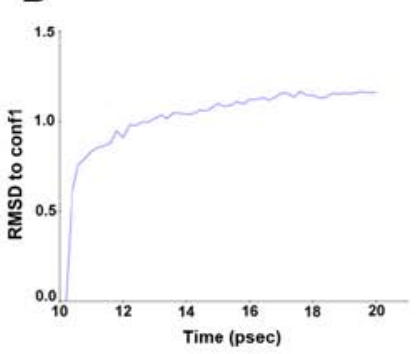

E

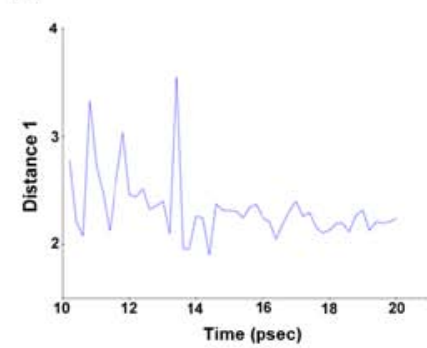

F

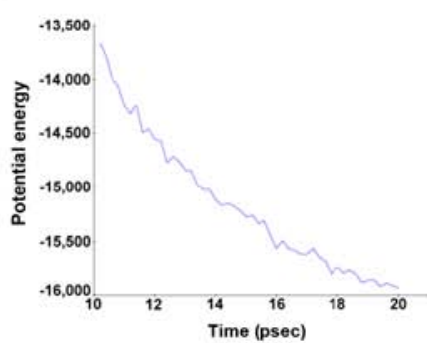

G

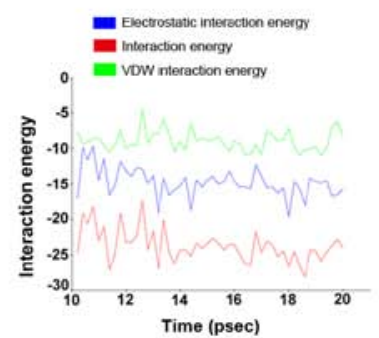

Figure 3. Protein-ligand interaction and molecular dynamic simulation of HNE and CAPE. (A) Interaction models of HNE and CAPE in the best docking pose. (B) Detailed interaction modes of HNE and CAPE in the best docking pose. (C) Model of HNE and CAPE in solvent. (D) Drug positional RMSD. (E) Distance between the O of CAPE and HN in the amino residue of ARG178 in HNE. (F) Potential energy of amino residue group between HNE and CAPE. (G) Interaction energy of amino residue group between HNE and CAPE through all the molecular dynamics. CAPE is in stick representation, while the residues of HNE are presented by solid style in line representation. Hydrogen bonds are depicted by green dotted lines. Water is depicted by pink. $\mathrm{C}, \mathrm{H}$ and $\mathrm{O}$ are colored in gray, white and red, respectively.

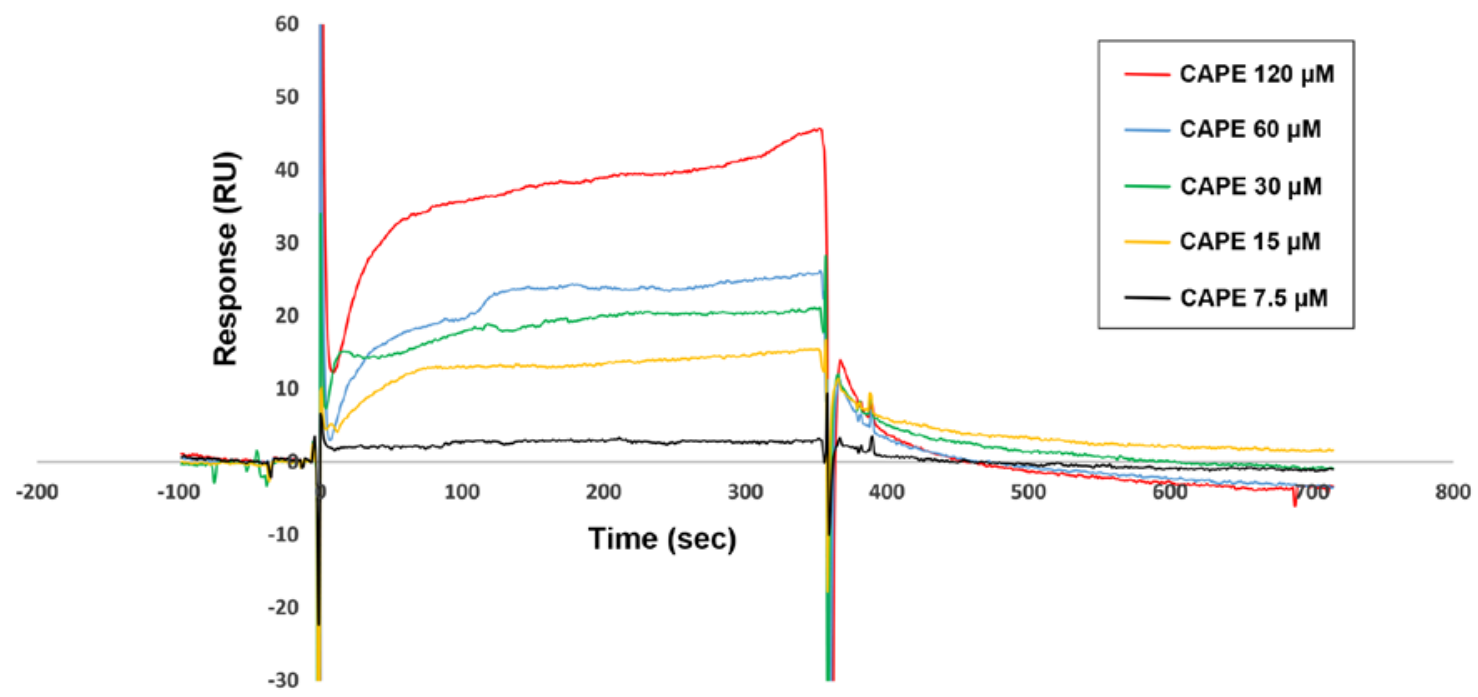

Figure 4. Binding affinity studies of HNE and CAPE. Real-time binding affinity data of CAPE (120 $\mu \mathrm{M}$ with 2-fold dilutions down to $7.5 \mu \mathrm{M})$ over the immobilized HNE surface on the CM5 chip.

$\alpha 1$-antitrypsin was regulated by CAPE in PANC-1 cells. As shown in Fig. 2D-F, with or without HNE, CAPE (18-60 $\mu \mathrm{M})$ did not modulate $\alpha 1$-antitrypsin expression at either the transcriptional or translational level.

hNE is a direct protein-target for CAPE in silico. We measured the specific binding of CAPE to HNE using molecular dynamics. The in silico drug target docking modeling analysis indicated that CAPE directly bound to the binding pocket of HNE (Fig. 3A-C) with $25.6581 \mathrm{kcal} / \mathrm{mol}$ in its best binding confirmation. As shown in Fig. 3B, ARG178, CYS168 and ARG217 play decisive roles in H-bond formation, which contributes to stabilizing the complex of HNE and CAPE. RMSD reference of CAPE, plotted in Fig. 3D, indicated that the interaction of the receptor-ligand complex reached equilibrium state after 14 psec. Similar results were obtained 
from interaction analysis between the $\mathrm{O}$ of CAPE and HN in the amino residue of ARG178 in HNE (Fig. 3E-G). Thus, the residue of the catalytic site stabilizes the interaction between CAPE and HNE.

CAPE directly binds to HNE according to SPR analysis. We determined the binding affinity of CAPE for HNE based on SPR. Fig. 4 shows that the response units were significantly increased with increasing CAPE (7.5-120 $\mu \mathrm{M})$, indicating that CAPE was able to directly bind to HNE in a concentration-dependent manner. $\mathrm{K}_{\mathrm{a}}$ and $\mathrm{K}_{\mathrm{d}}$ values for CAPE binding to immobilized HNE on the CM5 chip were $1.97 \times 10^{5} \mathrm{M}^{-1} \mathrm{sec}^{-1}$ and $2.35 \times 10^{-2} \mathrm{sec}^{-1}$, respectively. The equilibrium dissociation constant $\left(\mathrm{KD}=\mathrm{K}_{\mathrm{d}} / \mathrm{K}_{\mathrm{a}}\right)$ was $1.19 \times 10^{-7} \mathrm{M}$. Thus, HNE is a direct target of CAPE.

\section{Discussion}

We investigated the anti-migratory and anti-growth effects of CAPE on HNE-induced PANC-1 cells. Using an elastase assay, SPR and computer-aided drug design (CADD), we analyzed the interaction between CAPE and HNE. Data showed that CAPE inhibited the migration and viability of the HNE-induced PANC-1 cells. In addition, CAPE solely and directly interacts with HNE.

CAPE, a polyphenolic natural product and active component of propolis, has been reported to inhibit tumor proliferation and metastasis (23), as well as induce the apoptosis of various cancer cells in vitro and in vivo, including central nervous system, gastrointestinal system and breast cancer as well as leukemia $(18,24)$. CAPE has a range of molecular targets and influences numerous biochemical and molecular processes and it is documented to be safe for humans. Tumor cell lines are significantly more sensitive to CAPE than non-cancerous cells (19). An in vivo study demonstrated that propolis has little acute oral toxicity ( $\mathrm{LD}_{50} 2,000-7,300 \mathrm{~g} / \mathrm{kg}$ for mice) (18). In addition to its antitumor effect, CAPE also is an anti-inflammatory, antioxidant compound with neuroprotective and immune modulatory functions (16).

Many molecules are involved in the antitumor activity of CAPE, including NF- $\kappa \mathrm{B}, \mathrm{JNK}$, caspase-3, activator protein-1 (AP-1) and tumor necrosis factor (TNF). Cancer progression is a complex process and its pathogenic mechanism remains somewhat elusive, thus CAPE is considered to be a multitarget natural compound. That CAPE may inhibit tumor development via other mechanisms cannot be excluded. Thus, we investigated the novel inflammation-related mechanisms of CAPE involved in its antitumor activity in pancreatic cancer.

There is a growing awareness that inflammation is an important risk factor for the development of cancer at many sites, such as the lung, bladder, gastrointestinal tract, skin and vulva (12). When Virchow observed the presence of inflammatory cells within neoplastic tissue in the 19th century, the link between inflammation and cancer was first suggested, but the mechanism underlying this is only recently being clarified $(12,25)$. Inflammation plays a role in promoting cancer growth, tissue invasion and metastasis, due to reactive oxygen species and the induction of cell cycling. Cancer arising in a background of inflammation may therefore be associated with a worse prognosis (13).
PDAC is a lethal cancer with a poor prognosis. In addition, a lack of early symptoms and distant metastatic spread by the time of diagnosis and the intrinsic and acquired resistance to conventional therapeutic modalities makes this a difficult cancer to treat (26). Cellular mechanisms contributing to pancreatic cancer development and progression are undefined but inflammation is thought to be key particularly in the context of repeated acute pancreatic injury. Acute pancreatitis (AP) is a clinical syndrome which begins with acute injury to the pancreas and injurious substances include alcohol, smoking, gall stones, drugs and obesity. Recurrent acute pancreatic insult can lead to chronic pancreatitis, which is a strong risk factor for pancreatic cancer (27).

HNE degrades phagocytosed foreign organic molecules within cells. Extracellular HNE can degrade various extracellular proteins and induce production of inflammatory cytokines from epithelial cells (28). Several studies indicate that HNE concentration and activity are elevated in AP, and are reliable markers of the severity of AP. Specific HNE inhibitors, such as sivelestat, have potential for treating AP (29). Sivelestat is the first drug applied to systemic inflammatory response syndrome (SIRS) and sivelestat may suppress breast and esophageal cancer and cholangiocarcinoma and gastric carcinoma (30-33).

Previously, we reported that curcumin can inhibit HNE with a similar CDOCKER interaction energy to that of CAPE. However, the equilibrium dissociation constant $\left(\mathrm{KD}=\mathrm{K}_{\mathrm{d}} / \mathrm{K}_{\mathrm{a}}\right)$ of CAPE binding to $\mathrm{HNE}\left(1.19 \times 10^{-7} \mathrm{M}\right)$ was significantly lower than that of curcumin $\left(3.17 \times 10^{-5} \mathrm{M}\right)$ based on SPR. Thus, CAPE could bind to HNE better than curcumin $(21,22)$. In addition, many other natural compounds have been screened as HNE inhibitors, including flavonoids, caffeic acid derivatives, phenolics, monoterpenes, sesquiterpenes, triterpenes and long-chain fatty acids (11).

Traditional approaches to drug discovery rely on screening of numerous compounds to identify a potential candidate, which is an expensive and time consuming process. CADD approaches have been widely employed to identify lead compounds and optimize drug development against various targets (34). CADD uses rational drug design methods, but biological studies are necessary to verify CADD data. SPR is ideal for generating reliable data concerning interactions between biomolecules and small ligands with real-time analysis and detection of low-affinity target binders (35). CADD and SPR provide a fast, inexpensive, and reliable method of drug discovery.

In conclusion, CAPE modulates tumor migration and growth induced by HNE and CAPE blocks HNE activity directly interacting with HNE. These data offer a new putative mechanism of CAPE activity against tumor migration and growth and suggest that CAPE may be a potential anticancer drug.

\section{Acknowledgements}

The present study was supported by the National Natural Science Foundation of China (nos. 81473235, 81673453, 91129727, 81673486, 81270049 and 81373405), and the Research Fund from the Ministry of Education of China (111 Projects no. B07001). 


\section{References}

1. Paulson AS, Tran Cao HS, Tempero MA and Lowy AM: Therapeutic advances in pancreatic cancer. Gastroenterology 144 1316-1326, 2013

2. Siegel R, Naishadham D and Jemal A: Cancer statistics, 2012. CA Cancer J Clin 62: 10-29, 2012.

3. Siegel R, Naishadham D and Jemal A: Cancer statistics, 2013. CA Cancer J Clin 63: 11-30, 2013.

4. Rahib L, Smith BD, Aizenberg R, Rosenzweig AB, Fleshman JM and Matrisian LM: Projecting cancer incidence and deaths to 2030: The unexpected burden of thyroid, liver, and pancreas cancers in the United States. Cancer Res 74: 2913-2921, 2014.

5. Reid MD, Basturk O, Thirabanjasak D, Hruban RH, Klimstra DS Bagci P, Altinel D and Adsay V: Tumor-infiltrating neutrophils in pancreatic neoplasia. Mod Pathol 24: 1612-1619, 2011.

6. Gaida MM, Steffen TG, Günther F, Tschaharganeh DF, Felix K, Bergmann F, Schirmacher P and Hänsch GM: Polymorphonuclear neutrophils promote dyshesion of tumor cells and elastasemediated degradation of E-cadherin in pancreatic tumors. Eur J Immunol 42: 3369-3380, 2012

7. Sköld CM, Liu X, Umino T, Zhu Y, Ohkuni Y, Romberger DJ, Spurzem JR, Heires AJ and Rennard SI: Human neutrophil elastase augments fibroblast-mediated contraction of released collagen gels. Am J Respir Crit Care Med 159: 1138-1146, 1999.

8. Kouakou-Siransy G, Sahpaz S, Nguessan GI, Datté JY, Brou JK, Gressier B and Bailleul F: Effects of Alchornea cordifolia on elastase and superoxide anion produced by human neutrophils. Pharm Biol 48: 128-133, 2010.

9. Sato T, Takahashi S, Mizumoto T, Harao M, Akizuki M, Takasugi M, Fukutomi T and Yamashita J: Neutrophil elastase and cancer. Surg Oncol 15: 217-222, 2006.

10. Lucas SD, Costa E, Guedes RC and Moreira R: Targeting COPD: Advances on low-molecular-weight inhibitors of human neutrophil elastase. Med Res Rev 33 (Suppl 1): E73-E101, 2013.

11. Siedle B, Hrenn A and Merfort I: Natural compounds as inhibitors of human neutrophil elastase. Planta Med 73: 401-420, 2007.

12. McKay CJ, Glen P and McMillan DC: Chronic inflammation and pancreatic cancer. Best Pract Res Clin Gastroenterol 22: 65-73, 2008 .

13. Greer JB and Whitcomb DC: Inflammation and pancreatic cancer: An evidence-based review. Curr Opin Pharmacol 9: 411-418, 2009.

14. Sun Z and Yang P: Role of imbalance between neutrophil elastase and alpha 1-antitrypsin in cancer development and progression. Lancet Oncol 5: 182-190, 2004.

15. Kamohara H, Sakamoto K, Mita S, An XY and Ogawa M: Neutrophil elastase inhibitor (ONO-5046.Na) suppresses the proliferation, motility and chemotaxis of a pancreatic carcinoma cell line, Capan-1. Res Commun Mol Pathol Pharmacol 98: 103-108, 1997.

16. Tolba MF, Azab SS, Khalifa AE, Abdel-Rahman SZ and Abdel-Naim AB: Caffeic acid phenethyl ester, a promising component of propolis with a plethora of biological activities: A review on its anti-inflammatory, neuroprotective, hepatoprotective, and cardioprotective effects. IUBMB Life 65: 699-709, 2013.

17. Chan GC, Cheung KW and Sze DMP: The immunomodulatory and anticancer properties of propolis. Clin Rev Allergy Immunol 44: 262-273, 2013.

18. Akyol S, Ozturk G, Ginis Z, Armutcu F, Yigitoglu MR and Akyol O: In vivo and in vitro antineoplastic actions of caffeic acid phenethyl ester (CAPE): Therapeutic perspectives. Nutr Cancer 65: 515-526, 2013.
19. Watanabe MA, Amarante MK, Conti BJ and Sforcin JM: Cytotoxic constituents of propolis inducing anticancer effects: A review. J Pharm Pharmacol 63: 1378-1386, 2011.

20. Melzig MF, Pertz HH and Krenn L: Anti-inflammatory and spasmolytic activity of extracts from Droserae herba. Phytomedicine 8: 225-229, 2001.

21. Fan S, Xu Y, Li X, Tie L, Pan Y and Li X: Opposite angiogenic outcome of curcumin against ischemia and Lewis lung cancer models: In silico, in vitro and in vivo studies. Biochim Biophys Acta 1842: 1742-1754, 2014.

22. Xu Y, Zhang J, Han J, Pan X, Cao Y, Guo H, Pan Y, An Y and $\mathrm{Li} \mathrm{X}$ : Curcumin inhibits tumor proliferation induced by neutrophil elastase through the upregulation of $\alpha 1$-antitrypsin in lung cancer. Mol Oncol 6: 405-417, 2012.

23. Chen MJ, Shih SC, Wang HY, Lin CC, Liu CY, Wang TE, $\mathrm{Chu} \mathrm{CH}$ and Chen YJ: Caffeic Acid phenethyl ester inhibits epithelial-mesenchymal transition of human pancreatic cancer cells. Evid Based Complement Alternat Med 2013: 270906, 2013.

24. Chen MJ, Chang WH, Lin CC, Liu CY, Wang TE, Chu CH, Shih SC and Chen YJ: Caffeic acid phenethyl ester induces apoptosis of human pancreatic cancer cells involving caspase and mitochondrial dysfunction. Pancreatology 8: 566-576, 2008.

25. Hausmann S, Kong B, Michalski C, Erkan M and Friess H: The role of inflammation in pancreatic cancer. Adv Exp Med Biol 816: 129-151, 2014

26. Momi N, Kaur S, Krishn SR and Batra SK: Discovering the route from inflammation to pancreatic cancer. Minerva Gastroenterol Dietol 58: 283-297, 2012.

27. Kolodecik T, Shugrue C, Ashat M and Thrower EC: Risk factors for pancreatic cancer: Underlying mechanisms and potential targets. Front Physiol 4: 415, 2014.

28. Aikawa $\mathrm{N}$ and Kawasaki Y: Clinical utility of the neutrophil elastase inhibitor sivelestat for the treatment of acute respiratory distress syndrome. Ther Clin Risk Manag 10: 621-629, 2014.

29. Novovic S, Andersen AM, Nord M, Astrand M, Ottosson T, Jørgensen LN and Hansen MB: Activity of neutrophil elastase reflects the progression of acute pancreatitis. Scand J Clin Lab Invest 73: 485-493, 2013.

30. Nawa M, Osada S, Morimitsu K, Nonaka K, Futamura M, Kawaguchi Y and Yoshida K: Growth effect of neutrophil elastase on breast cancer: Favorable action of sivelestat and application to anti-HER2 therapy. Anticancer Res 32: 13-19, 2012.

31. Nishiyama J, Matsuda M, Ando S, Hirasawa M, Suzuki T and Makuuchi $\mathrm{H}$ : The effects of the early administration of sivelestat sodium, a selective neutrophil elastase inhibitor, on the postoperative course after radical surgery for esophageal cancer. Surg Today 42: 659-665, 2012.

32. Hanada N, Kusano S, Hori K and Momi H: Good response in ARDS treated with sivelestat sodium hydrate during chemotherapy for cholangiocarcinoma. Gan To Kagaku Ryoho 34: 1303-1306, 2007 (In Japanese).

33. Wada Y, Yoshida K, Hihara J, Konishi K, Tanabe K, Ukon K, Taomoto J, Suzuki T and Mizuiri H: Sivelestat, a specific neutrophil elastase inhibitor, suppresses the growth of gastric carcinoma cells by preventing the release of transforming growth factor-alpha. Cancer Sci 97: 1037-1043, 2006.

34. Aparoy P, Reddy KK and Reddanna P: Structure and ligand based drug design strategies in the development of novel 5-LOX inhibitors. Curr Med Chem 19: 3763-3778, 2012.

35. Crauste C, Willand N, Villemagne B, Flipo M, Willery E, Carette X, Dimala MM, Drucbert AS, Danze PM, Deprez B, et al: Unconventional surface plasmon resonance signals reveal quantitative inhibition of transcriptional repressor EthR by synthetic ligands. Anal Biochem 452: 54-66, 2014. 